\title{
A methodological and physical instrumentaion to support experimentation in telE-learning
}

\author{
Aude Dufresne, France Henri and Richard Hotte \\ LICEF Research Centre, Télé-université of Québec, Canada
}

\begin{abstract}
This article is an attempt to promote a research and development methodology that is action-oriented in order to improve the development of telE-learning environments and software artefacts fully adapted to learning activities. We start by presenting an overview of the two main phases of action-oriented research, stressing the importance of control methods. Then we describe difficulties in applying these methods and, finally, we introduce the LORIT, a recent initiative intended to support instrumentation in tele-learning research.
\end{abstract}

Key words: activity theory, learning technology, oriented-action research, research methodology, telE-learning

\section{INTRODUCTION}

\subsection{Research practices in distance education}

The emergence of a new mode of distance learning has stimulated research activities and has mobilised important human and economic investments for the development of technological environments to support what is called e-learning, e-training or tele-learning. Research contributions from various fields such as the cognitive sciences, cognitive and pedagogical engineering, and software engineering have contributed to the development of learning environments and systems and to the understanding of the learning processes involved. Methodologies used for distance education research have not had as strong an impact as one might have expected due to their limitations. 
Many authors who have examined the research literature in distance education (see Dillon and Gunawardena, 1992; Saba, 2000, Perraton, 2000; Berge and Mrozowski, 2001) have criticised it for showing limitations in the conceptualisation of the domain and of research questions, for suffering from a lack or absence of theory, and for having been dominated by descriptive research and comparative studies. They observed that an important number of research projects compare distance education to face-to-face instruction trying to verify the effectiveness of one mode over the other, or to measure the relative effectiveness of one distance delivery media over another.

However, as Saba (2001) explains, in recent years, distance education researchers have adopted new research approaches moving from theoretical and experimental comparative studies to methodological approaches, allowing for the capture of a wider spectrum of empirical data on interaction in both its qualitative and quantitative format. Their studies tend to focus on a small number of subjects in order to take a deeper look at the interaction between teacher, tutor or trainer, and learner. This paper contributes to the reflection on a new research approach in the field of distance education.

\subsection{Toward a new conceptualisation of research in the domain}

In the field of distance education, the trend of research seems to be similar to what can be observed in educational research where naturalistic approaches, such as ethnography and action research, are applied to develop a better understanding of the social nature of the relationships among actors involved in the learning process, and to contribute to both the development of technology and the design of its use (Nulden, 2001). Research themes are more and more focused on the learner and on interaction, indicating its centrality to the process of learning and teaching in conceptualising the process of teaching and learning (Saba, 2000; Berge and Mrozowski, 2001). With the development of information and communication technologies and the emergence of contemporary theories of education, media and technologies are seen more and more as support to the learning process. Distance is not conceived any longer as a limitation, but as a situation which can be viewed positively, and where media and technologies, with their attributes including interactive capacities, are exploited to stimulate, enrich and support the learning process and the networking of learners.

Inspired by a vision of distance education which includes tele-learning practices, new research questions are formulated and research methodology is opening up to naturalistic approaches. This article is an attempt to promote research and development methodology that is action-oriented in order to 
improve the development of tele-learning environments and artefacts fully adapted to learning activities.

\section{ACTION-ORIENTED RESEARCH}

Aiming at the development of tele-learning capacities and the production of learning environments composed of instrumental software artefacts, action-oriented research is characterised by interventions closely related to reality (action) and by the interaction between researchers and actors, between knowledge building and action. This approach favours the integration of participative design and is characterised by a back and forth process. In this context, the production of virtual learning environments becomes the research object, and the methodological approach is based on a temporal and logical organisation of operations related to two major phases as shown in Figure 1.

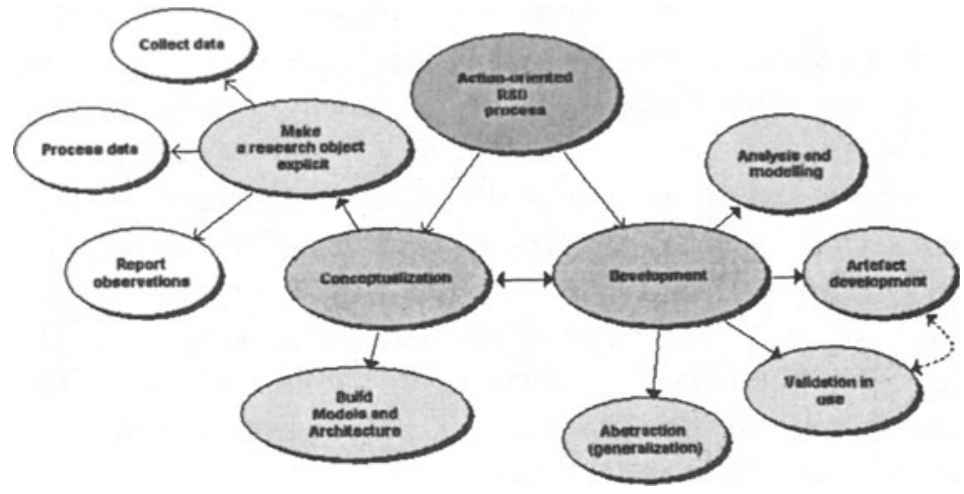

Figure 1. Action-oriented research and development approach

- The conceptualisation phase is characterised by an effort to make the research object explicit, and by an understanding and a design of activity related to the research object using theories, data collection and process analysis.

- The development of the various software components of the system includes four main steps - analysis, development, validation in use, and abstraction - in order to determine the reusability of the artefact.

During the first phase, the researcher utilises social science methods, such as field surveys, to deepen the perception of the actor's activity and of the context in which the system to be developed will be used. Also, pedagogical engineering methods are useful to generate models such as 
activity models of the actor, and models of the tele-learning environment itself. To conduct the second phase, software engineering methods and techniques guide the development of the systems' components. Throughout the two phases, participative design allows the participation of the user in all steps of the design, development and evaluation processes (Nardi, 1997). This action-oriented approach is characterised by the integration of a validation in use step to measure the usability and the utility of the system for the end user.

\subsection{Transversal and iterative control methods}

To ensure rigorous research, control methods such as experimentation, validation and evaluation are introduced in the research methodology and applied to the conceptualisation as well as the development phases. These methods generate results that enable the researcher to work iteratively towards a system or an artefact well adapted to the context of its use. Experimentation consists of provoking an observation in order to study a phenomenon. The data collection provides understanding of new situations and description of specific human behaviours. It can be conducted in natural or artificial settings, using naturalistic or comparative experimental schemes. Validation is an operation conducted with the intention to verify the correspondence of an object with its model or its representation (Legendre, 1993). Generally speaking, evaluation is the process by which the quality of the artefact is verified according to current practices or established standards. Evaluation leads to a judgment or to decision-making. This judgment, qualitative or quantitative, is obtained by comparing the observable characteristics with explicit criteria derived from standards (Legendre, 1993).

These three control methods are transversal and iterative to the research process. This means that the system under development can be experimented, validated and evaluated both during conceptualisation and development phases, and that considerable back and forth movement can exist between the two phases. One can return back to a previous step or the previous phase to improve the artefact in accordance with the observed results.

\subsection{Constraints related to the implementation of control methods}

The delivery of coherent systems capable of satisfying the needs of the user by supporting adequately the individual's learning activities raises a 
series of issues, such as epistemological questions, methodological difficulties and material problems. The current constraint in the field of telelearning is the weakness of the instrumentation of the activity of the researcher in terms of the three aspects mentioned above.

Research conducted in a natural setting imposes restrictions on the methodology. The research process itself is submitted to a series of constraints that limit the number and the scale of experimentation, validation and evaluation interventions required to understand the users' points of view, to observe their behaviours in the situation of use, to verify the ergonomics of the interfaces, and to evaluate constitutive elements. Since it is difficult, or otherwise impossible, in a natural and large-scale setting to implement experimentation, validation and evaluation at all phases of the research process, the solution is to work in an artificial setting, such as a laboratory, and on restricted groups.

\section{FROM PRINCIPLES TO REALITY}

It might be trivial to stress, as we did, the necessity to integrate control methods at all steps of an action-oriented research and development approach. But in practice, implementing them is not as easy, due to the costs implied, the time consumed and the rareness or non-existence of adequate technological infrastructure to conduct them. It is the case at the Téléuniversité (a higher education autonomous institution totally dedicated to distance education) where learning environments and artefacts developed by the LICEF Research Centre are often transferred and used in courses before they have reached sufficient maturity. They are tried out in real situations, involving real groups of students and real tutors. They are submitted to regular academic management operations and integrated into the delivery system of the institution. This situation raises ethical and methodological issues and creates unnecessary stress. For the students, the quality of learning and learning time could be compromised by cognitive overload. For the tutor, professional issues could be linked to working conditions. Also, academics and technological management staff should not be involved in experimenting an innovation. Finally, from a methodological point of view, experiments conducted under such uncertain and risky conditions cannot pretend to be without bias and are a poor exploiter of the natural setting.

As we have explained earlier, experimentation, validation and evaluation as steps of a research methodology have to be conducted in a protected setting, such as a laboratory, before the product is implemented by an institution. Dimensions of the system such as the pedagogical scenario and interface as well as computer design and programming, can be experimented 
with, validated and evaluated under laboratory conditions. Direct synchronous laboratory observation should be conducted until the research object has reached a sufficient level of maturity that it will not jeopardise the learning process nor the professional integrity of the various actors involved. In order to strengthen their general research and development methodology, researchers from the LICEF Research Centre have decided to conduct more rigorous and extensive experimentation, validation and evaluation before any of their artefacts are integrated in real settings.

\section{IMPROVING METHODOLOGIES}

The laboratory observatory for research in tele-learning engineering (LORIT) was developed at the LICEF Research Centre to fulfil the need for more controlled observation, validation and experimentation of tele-learning systems. It has been designed to test advanced technologies and to collect and store experimental data in a structured manner to facilitate the compilation and processing of results. The LORIT offers 10 multifunctional stations, plus three remote stations for observation and control, as well as various external connections and recording facilities.

With these facilities, the LORIT can be used to validate, experiment with and evaluate a system from different perspectives - technical, pedagogical, ergonomic. It may be useful at the two phases of conceptualisation and development of tele-learning systems: experimentation and observation of existing systems, design and validation of software artefacts, experimentation and evaluation of parts of or the whole of a new system.

\subsection{Using scenarios to structure action-oriented research}

As suggested by action-oriented research and user-centred design, generic scenarios were developed at the LORIT to help to structure various tests. These scenarios are partly inspired by the tele-learning models described by Paquette (2000): distributed class, web self-training, collaborative learning, learning communities, and performance support systems. For each scenario, a specific set-up is defined, which can be adapted for specific cases.

For instance, for validation of a system designed to support distributed class scenarios, the remote facility of the LORIT can be used to simulate distant teacher-student interaction by isolating learners in a room while the teacher is in the teaching room. For evaluation purposes, the control room, the server and the video bridge may be used to interact in a real distance mode with distant learners, and for recording some of the interactions. 
In order to validate the collaborative capacities of a system, stations can be isolated and connected using videoconferencing or MS NetMeeting (see Figure 2). Even asynchronous communication can be studied while students are logging on to the system from outside (via a computer log, and audio comments using the bridge).

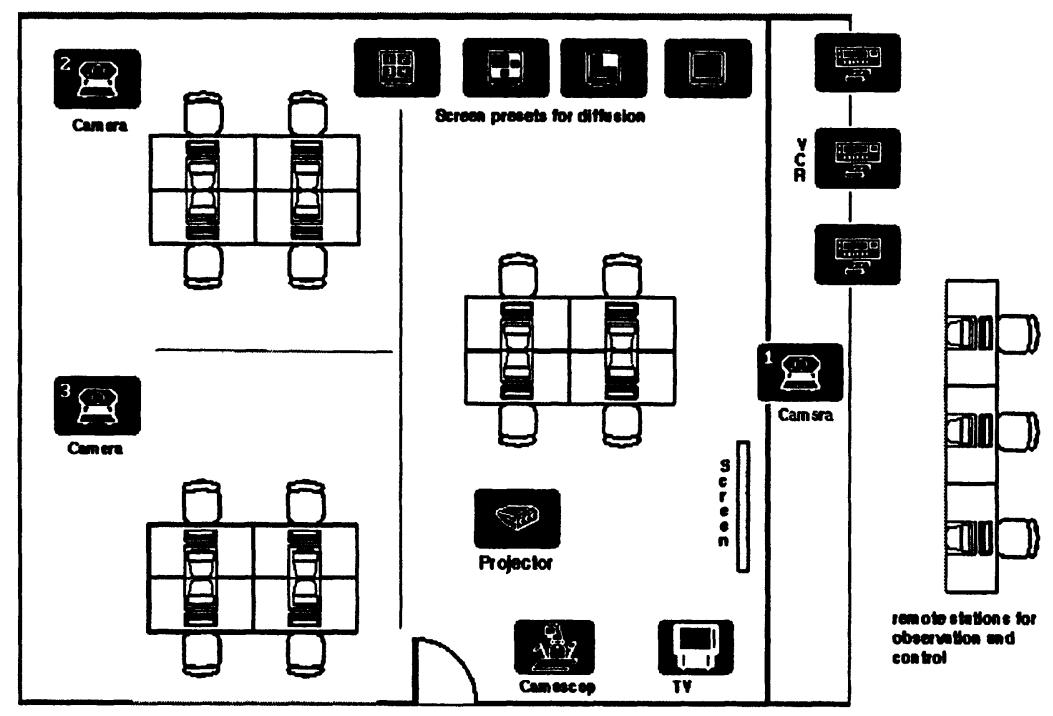

Figure 2. Example of a set-up of the LORIT to validate collaborative scenario

Other scenarios include:

- experimental testing of components of systems for technical, theoretical or evaluation purposes;

- ergonomic evaluation with talk-aloud protocols while users interact with systems;

- 'wizard of Oz' techniques, where a coach in the remote station controls the system or tries to support a learner, in order to investigate support and how human interaction could be computerised in systems;

- brain storming and focus groups in conceptualisation phases or following experimentation of systems can also take advantage of the LORIT recording infrastructure.

\section{CONCLUSION}

With this infrastructure, new models of experimentation, validation and evaluation of tele-learning processes are made possible, using a more 
naturalistic approach, centred around activity-based scenarios. Thus in the LORIT controlled environment, systems and tele-learning approaches may be analysed and tested in an iterative way during conceptualisation as well as development phases. It may also be used to collect data for the final evaluation of systems. The possibility to make finer analysis of the use of systems should improve our understanding of how they should be made. It should help protect and prepare the delivery of the products of research into the field. Finally, it should improve our understanding of the processes of tele-learning.

\section{REFERENCES}

Berge, L.B. and Mrozowski, S. (2001) Review of Research in Distance Education. The American Journal of Distance Education, 15, 3, 5-19

Bødker, S. (1999) Scenarios in User-Centred Design - setting the stage for reflection and action. Proceedings of the 32nd Hawaii International Conference on System Sciences, IEEE.

Dillon, C. and Gunawardena, C. (1992) Evaluation research in distance education. British Journal of Education, 23, 3, 181-194

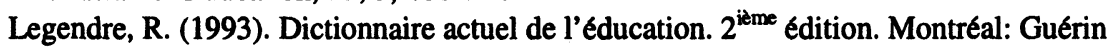

Nardi, B.-A. (1997) Context and Consciousness. Activity Theory and Human-Computer Interaction. Cambridge: The MIT Press

Nulden, U. (2001) e-education: research and practice. Journal of Computer Assisted Learning, $17,4,363-375$

Paquette, G. (2000) Construction de portails de téléapprentissage. Explora - une diversité de modèles pédagogiques. Sciences et techniques éducatives, 7, 1/2000, 207-226

Perraton, H. (2000) Rethinking the Research Agenda. International Review of Research in Open and Distance Learning, 1, 1

Saba, F. (2000). Research in Distance Education: A Status Report. International Review of Research in Open and Distance Learning, 1, 1

\section{BIOGRAPHIES}

Aude Dufresne is with the Communication Department at the Université de Montréal. She is also a researcher at the LICEF Research Centre. France Henri and Richard Hotte are both with the Science and Technology Department at the Télé-université of Québec and researchers at the LICEF Research Centre. 